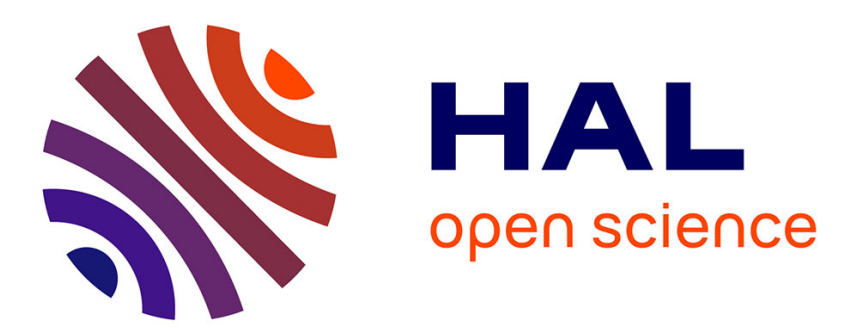

\title{
A first approach for a passenger-centered behavior on driverless vehicles
}

\author{
David González, Francisco Navas, Imane Mahtout, Vicente Milanés
}

\section{To cite this version:}

David González, Francisco Navas, Imane Mahtout, Vicente Milanés. A first approach for a passengercentered behavior on driverless vehicles. MED'2020 - 28th Mediterranean Conference on Control and Automation, Sep 2020, Saint-Raphaël / Virtual, France. hal-03130132

\section{HAL Id: hal-03130132 \\ https://hal.inria.fr/hal-03130132}

Submitted on 3 Feb 2021

HAL is a multi-disciplinary open access archive for the deposit and dissemination of scientific research documents, whether they are published or not. The documents may come from teaching and research institutions in France or abroad, or from public or private research centers.
L'archive ouverte pluridisciplinaire $\mathbf{H A L}$, est destinée au dépôt et à la diffusion de documents scientifiques de niveau recherche, publiés ou non, émanant des établissements d'enseignement et de recherche français ou étrangers, des laboratoires publics ou privés. 


\title{
A first approach for a passenger-centered behavior on driverless vehicles
}

\author{
David González ${ }^{1}$, Francisco Navas ${ }^{1}$, Imane Mahtout $^{23}$ and Vicente Milanés ${ }^{2}$
}

\begin{abstract}
Driverless shuttles are becoming one of the most promising applications of automated vehicle technologies in the short term. For a real system deployment, passenger's acceptance on the shuttle performance will play a key role. This paper presents a passenger-centered control algorithm design for increasing passenger's ride quality. Controllers with different performance but properly following the reference are developed for lane-level autonomous navigation. The whole solution has been implemented and tested in a real vehicle, using a roundabout as use-case scenario, providing encouraging results.
\end{abstract}

Index Terms - Vehicle control, Passengers' comfort, Passengers' acceptance, driverless shuttles.

\section{INTRODUCTION}

Two main trends can be identified on the intelligent road transport systems field: 1) automated vehicles that are ready to share the road with manually driven vehicles. The driver is still in the loop as a safety system-i.e. Tesla AutoPilot system; and 2) driverless vehicles (or shuttles) that can be fully independent on limited driving areas normally reserved to these vehicles. Driverless shuttles are getting more and more attention because of their potential to be implemented in the short term. In contrast with automated vehicles, they are operating in well-known environments [1] and eventually with infrastructure adapted to their needs [2], leading to more controlled and easy-to-deploy environments.

The first implementation of driverless shuttles can be found in the late 90's in a long term parking application at Schiphol airport [3]. Since then, multiple deployments worldwide were carried out. It is worth mentioning the EUfunded projects (CyberCars2 or CityMobil2, among others) related to last-mile deployment based on the Cybernetic Transport Concept where novel urban transport systems were developed and tested in several EU cities [4], [5]. All these demonstrations showed the potential of such systems for providing a solution for both: controlled environments and crowded areas. The last milestone was the long-term demonstrations carried out within the framework of the CityMobil-2 project, where people across different European cities experienced such systems. First guests about what users are expecting from the new mobility systems can be found

\footnotetext{
${ }^{1}$ Authors are with AKKA Technologies, Guyancourt, France. $\quad$ david-enrique.gonzalez-bautista, francisco-martin.navas-matos\} dakka.eu

${ }^{2}$ Authors are with the Research Department, Renault SAS, Guyancourt, France. \{imane.mahtout, vicente.milanes crenault. com

${ }^{3}$ Imane Mahtout is with the Robotics and Intelligent Transportation Systems (RITS) Team, INRIA, Paris, France. \{imane.mahtout (inria.fr
}

in the interviews carried out during project demonstrations [6].

Driverless shuttles are expected to replace conventional human-driven vehicles, meaning that passengers can be either seating or standing. Having this in mind, passengers' comfort play a key role in system acceptance. Comfort is explicitly linked to the vehicle's accelerations by acting either on the longitudinal or lateral vehicle motion reference and control. Related to the longitudinal control dynamics, [7] proposes a three phase speed control strategy using an acceleration-based evaluation index as control strategy for improving passengers' comfort. A comparative analysis between Bezier-based speed profile generation and constant jerk approach is presented in [8] where a combined lateral and longitudinal acceleration evaluation is carried out. A temporal optimization framework for speed profile generation considering multiple parameters as computational time, comfort and safety is presented in [9]. Optimal control has also been used to link the comfort constraints to the control problem as in [10]. They designed a Model Predictive Controller (MPC) and a learning approach to include the human driving style in the optimization process. However this is only done for the longitudinal actions. In [11] reinforcement learning has been applied to infer drivers' actions and then apply them to automated driving. The authors argue that individual perception of comfort is different among drivers and the vehicle should adapt to each of them.

Even if lateral and longitudinal accelerations are intimately related, previous works have been mainly focused on the longitudinal dynamic and control, especially from a planning point of view. Once a trajectory is optimized, the path tracker must be able to follow that trajectory whereas minimizing lateral and speed errors.

This paper proposes a controller with comfort considerations, minimizing lateral actions and adapting the ego vehicle performance to users' requirements. It uses as input not only the trajectory reference but also the lane width. The control algorithm benefits from such information to minimize lateral and longitudinal accelerations, while also avoiding to go out of bounds. The problem is tackled from the control point of view instead of the path planning to guarantee a given performance level even when the control action is slow or loose. The developed control system has been embedded in a robotized vehicle and tested in roundabouts.

\section{PROBLEM DESCRIPTION}

Driverless shuttles' acceptance depends on their ability to provide a good ride-quality to the passenger. One of the main advantages of this novel transport systems is the 
passenger's capacity to benefit from the time on board for doing other activities [12]. This is strongly linked to the vehicle's performance when driving autonomously. Driverless shuttle functional architecture relies on several functional blocks (see [13] for further details). However, the ride-quality depends mainly on the overall acceleration and jerk felt in the vehicle. For a proper performance, longitudinal and lateral actions need to adapt these accelerations and jerk profiles, considering that the definition of comfort differ from person to person [14].

The ability of driving autonomously in a given turn is usually developed using a local path planner [15]. It considers curvature-related information and the desired lateral acceleration to provide a smooth trajectory. This trajectory can be adapted accordingly to the optimization criteria or the desired lateral acceleration. However, vehicle's response depends on the control algorithm. If it remains identical, the way in which it recovers from lateral deviations remains the same, minimizing the impact of that trajectory change. Additionally, these trajectory changes don't guarantee vehicle stability from the full system analysis perspective.

Having this in mind, this paper proposes a passengercentered multi-controller structure, guaranteeing proper path tracking with independence of the controller gains, allowing to modify the vehicle response to the passenger's desires. This is done by designing the lateral control system (see 1) to smooth as much as possible the proposed trajectory while taking into account the passenger preferences. The starting point will always be the most performant controller (i.e. the one with the best tracking capabilities), being able to modify controller gains accordingly to passengers' preferences.

\section{A. General assumptions}

The following assumptions are considered for the present work:

- Only the lateral controller design is addressed in this work. Speed is known and properly followed by the longitudinal controller (the maximum speed was of 10 $\mathrm{m} / \mathrm{s})$.

- A given speed is set for turning, such speed is expected to be achieved before entering the turn.

- A proper trajectory is provided as an input to the controller (i.e. next $\mathrm{x}, \mathrm{y}$ coordinates, reference speed or curvature among others).

- The road layout has always enough space for the vehicle dimensions.

- Lane limits are known, as well as obstacles.

- Road layout information is known in a given horizon to allow control response optimization.

\section{VEHICLE CONTROL}

The general control structure is based on a speeddependent feedback controller (see details in [16]), as it provides good tracking capabilities for a given reference trajectory, with a simple control structure. Fig. 1 presents the lateral vehicle control block diagram. It is based on the control minimization of the desired curvature provided by

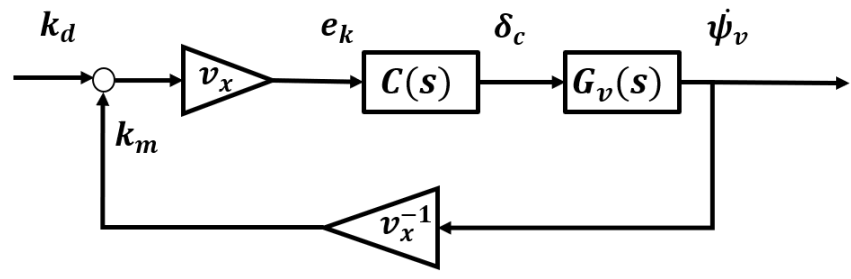

Fig. 1: Lateral vehicle control block diagram

the navigation stage $\left(k_{d}\right)$ and the current vehicle curvature $\left(k_{m}\right)$ calculated from the measured yaw rate $\left(\dot{\psi}_{v}\right)$ and the longitudinal vehicle speed $\left(v_{x}\right)$.

$$
C(s)=\frac{g}{s} \frac{\left(s^{2}+2 L s+2 L^{2}\right)}{2 L s^{2}}
$$

Equation 1 shows the controller transfer function $C$, where $g$ is the controller gain and $L$ is the inverse of the controller look-ahead time control point ( $L=1 /$ look-ahead-time, where the look-ahead-time can be described as the future point the vehicle aims to be with respect to the planned trajectory). It provides as output the desired steering wheel angle command $\left(\delta_{c}\right)$.

Multiple controllers have been developed with the goal of not only being able to perfectly track the center lane but also to benefit from the available lane width. The control point selection in look-ahead speed dependent controllers plays a key role that impacts the vehicle performance. To provide a passenger-centered design, a two step process was followed:

- A control objective with a phase margin over 30 degrees and a damping of 0.7 was set. This design criteria was applied in an optimization process up to finding the minimum look-ahead distance that guarantee such response.

- Controller gains were adapted to keep the system response for greater look-ahead values. This process was repeated up to the electronic horizon for each speed (in an offline optimization process), being able to provide a stable controller with the same response for each lookahead distance higher than the minimum one.

Figure 2 shows the Bode response for the different controllers. One can appreciate how the phase margin remains the same whereas the look-ahead distance increases. It reduces the closed loop system bandwidth, providing a slower response. In terms of passenger-centered approach, this can be seen as the trade-off between keeping a good control performance and increasing the comfort thanks to the lane width. It means that the vehicle will follow the trajectory as close as possible when applying the minimum look-ahead, being the faster controller. The higher the look-ahead, the smoother the response. The main idea is to reduce lateral acceleration, and consequently improve passengers' comfort and acceptability of the system.

\section{A. Passenger's acceptability considerations}

To have a passenger-oriented vehicle response, it is first necessary to define certain design parameters to include 


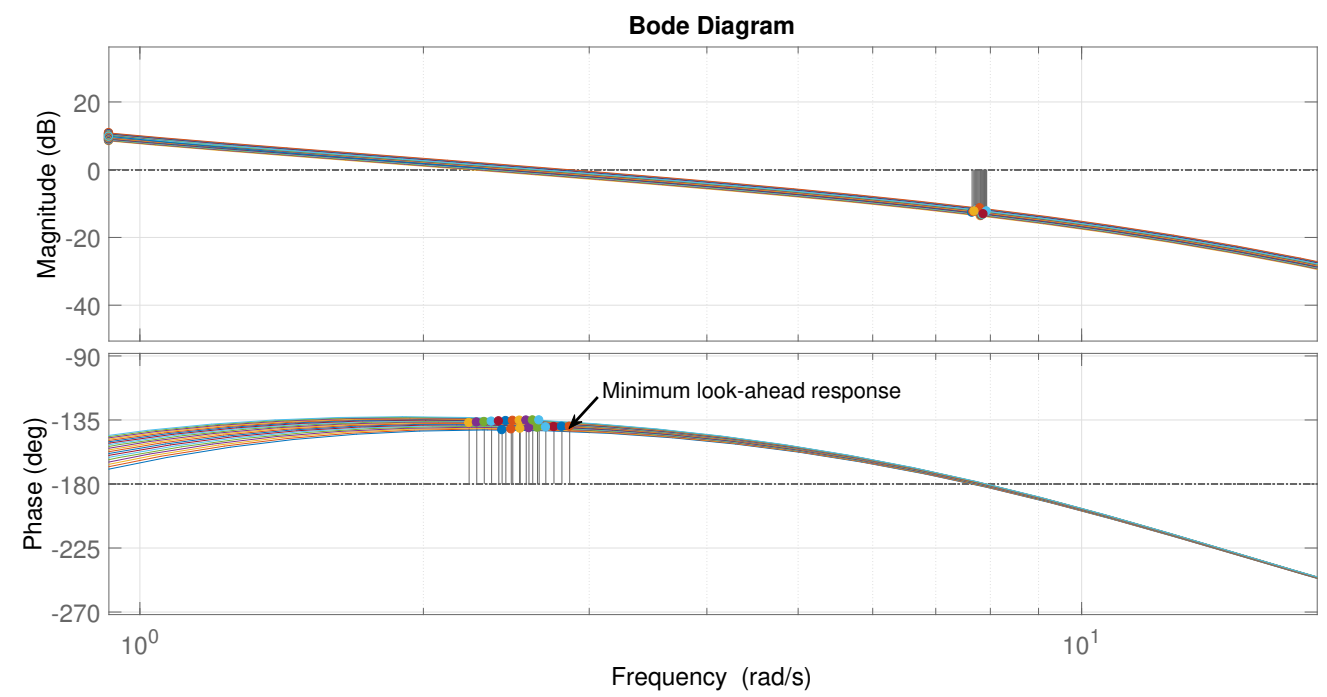

Fig. 2: Frequency response for different look-ahead time (from $0.8 \mathrm{~s}$ to $2.5 \mathrm{~s}$ ) at $10 \mathrm{~m} / \mathrm{s}$ with the same phase margin (from close-loop in Fig. 1).

safety and comfort. Those are focused in users' needs and expectations as follows:

- Lateral acceleration and steering wheel angle behavior as main design factors to follow the trajectory as humans do, reducing lateral accelerations in the path by profiting from the whole road width.

- Position of the vehicle in the road. The controller can be as smooth as possible, but the vehicle needs to always stay within the road boundaries.

\section{B. Physical trajectory constraints}

The optimization of the control response can be done up to the road boundaries. This means that the trajectory the vehicle will cover is safe and it will not encounter any curb or obstacles (we assume that the planned path of the vehicles is free from other road users and is dynamically feasible).

The dynamic (bicycle) vehicle model [17] can be used to this end, where future vehicle states can be predicted with different controllers. The vehicle model $\left(G_{v}\right)$ is as presented in Eq. 2:

$$
\begin{aligned}
\dot{X}_{v} & =A_{v} X_{v}+B_{v} u_{v} \\
Y_{v} & =C_{v} X_{v}
\end{aligned}
$$

where the state vector is:

$$
X_{v}=\left[\begin{array}{llll}
y_{v} & v_{y} & \psi_{v} & \dot{\psi}_{v}
\end{array}\right]^{T}
$$

$y_{v}, v_{y}, \psi_{v}, \dot{\psi}_{v}$ are the vehicle lateral position, lateral velocity, yaw angle and yaw rate respectively, $u_{v}=\delta_{c}$ is the steering angle in the front tire (assuming the rear wheels have zero steering angle). The system's matrices $A_{v}, B_{v}$ and $C_{v}$ are described below:

$$
A_{v}=\left[\begin{array}{cccc}
0 & 1 & 0 & 0 \\
0 & \frac{-\left(C_{f}+C_{r}\right)}{m v_{x}} & 0 & \frac{-a C_{f}+b C_{r}}{m v_{x}}-v_{x} \\
0 & 0 & 0 & 1 \\
0 & \frac{-\left(C_{f}+C_{r}\right)}{I_{z} v_{x}} & 0 & \frac{-a^{2} C_{f}+b^{2} C_{r}}{I_{z} v_{x}}
\end{array}\right]
$$

$$
\begin{gathered}
B_{v}=\left[\begin{array}{llll}
0 & \frac{C_{f}}{m} & 0 & \frac{a C_{f}}{I_{z}}
\end{array}\right]^{T} \\
C_{v}=\left[\begin{array}{llll}
0 & 0 & 0 & 1
\end{array}\right]
\end{gathered}
$$

where the longitudinal speed of the vehicle is denoted by $v_{x}, a$ and $b$ are the distances from the front and rear wheels respectively to the vehicle's center of gravity, $m$ is the mass of the vehicle and, $C_{f}$ and $C_{r}$ are the front and rear cornering stiffness respectively. Finally, the trajectory of the vehicle is given by $\mathrm{Eq}$. 4:

$$
\begin{aligned}
& \dot{x}=v_{x} \cos \left(\psi_{v}\right)-v_{y} \sin \left(\psi_{v}\right) \\
& \dot{y}=v_{x} \sin \left(\psi_{v}\right)+v_{y} \cos \left(\psi_{v}\right)
\end{aligned}
$$

where $\dot{x}$ and $\dot{y}$ are the global coordinates speeds.

The simulated future vehicle states predict the trajectory the real vehicle will cover per each controller configuration. These states can be evaluated, up to a horizon, to determine if any of them violates the boundaries of the road. The problem can be defined as to avoid the group of manifolds in which the vehicle overlaps any obstacle [18]. With a small modification, it is defined as in Eq. 5:

$$
\mathcal{C}_{\text {free }}=\left\{q \in \mathcal{C} \mid \mathcal{A}_{(q)} \cap \mathcal{O}=\emptyset\right\}
$$

where $\mathcal{C}_{\text {free }}$ is the set of all vehicle configurations $q=$ $(x, y, \psi)$ where $\mathrm{A}$ is without collision. $\mathcal{C}$ is the total configuration space, where $\mathcal{C}_{\text {free }} \subseteq \mathcal{C}, \mathcal{A}$ and $\mathcal{O}$ are the ego-vehicle and obstacles rigid bodies respectively, and $q$ represents the configurations of $\mathcal{A}$.

To verify this, the vehicle rigid body $\mathcal{A}$ can be represented as a 2-D bounding box, the road boundaries $\mathcal{O}$ can be described as a convex polygon formed from the lane boundaries information (i.e. a digital map for long enough road layout information is considered) and $q$ is represented by the simulated closed loop vehicle states while following the trajectory. Figure 3 gives a graphical representation of 


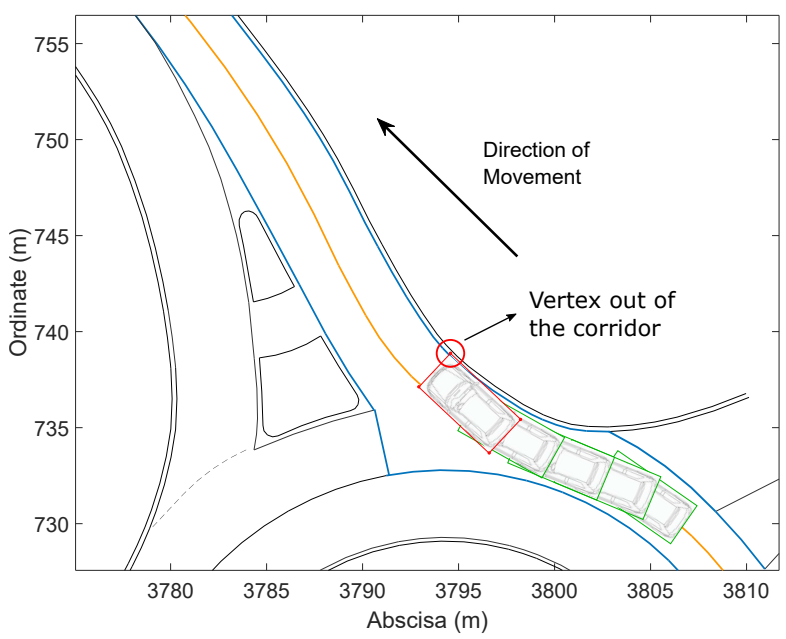

Fig. 3: The obstacle free area of the current roundabout path is presented in blue, the planned trajectory in yellow, soft and hard limits are in black.

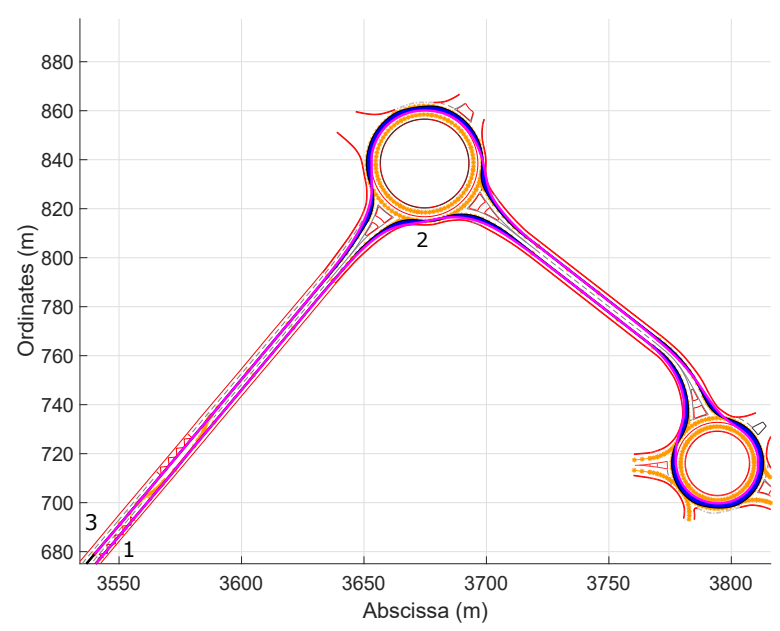

Fig. 4: Bird view of the simulation scenario. The itinerary started at point 1 , passing through point 2 , both roundabouts and finally reaching point three.

the verification (each vehicle position represents a state), which is done by solving the point-into-polygon problem. Boundaries are given in blue whereas the road center-lane is depicted in yellow. The vehicle model is evolved through time to find the set of $\mathcal{A}_{(q)}$ configurations that comply with Eq. 5.

Each of the bounding box vertices are evaluated to be within the road boundaries polygon. The evaluation is done by making the projection of each vertex to a sufficiently far away point and counting the number of intersections against each of the polygon segments. If the intersection number is odd, the vertex remains within the polygon (see green bounding boxes in Fig. 3) and is outside in case of no intersection or a pair number (represented in a red bounding box, indicating a violation of the boundaries).

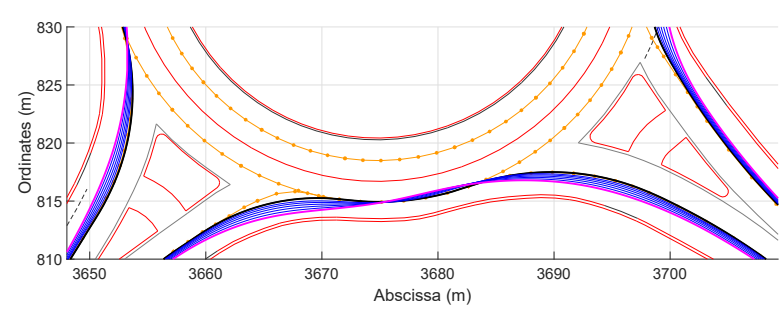

Fig. 5: Zoomed region 2 from Fig. 4

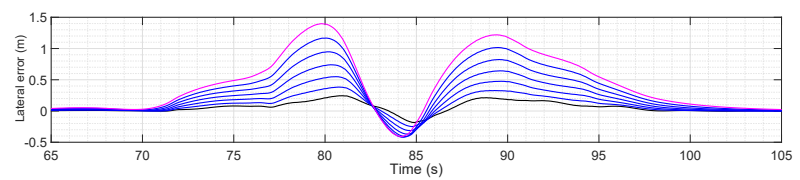

(a) Lateral error

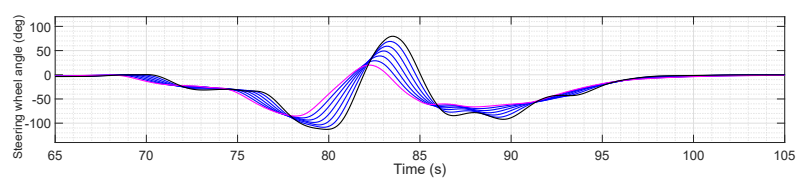

(b) Steering wheel angle response

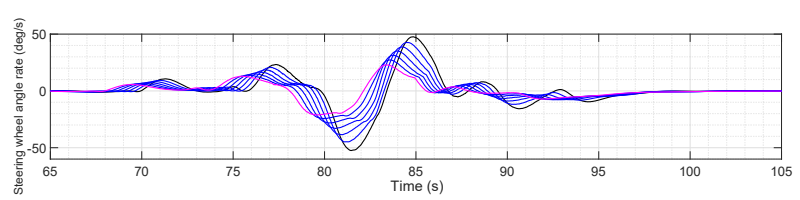

(c) Steering wheel angle rate

Fig. 6: Lateral error, steering wheel angle and rate for each controller configuration. The most restrictive controller configuration is depicted in black and the most relaxed one is presented in magenta. Other in-between controllers are depicted in blue.

\section{Simulation results}

Simulation scenario is composed of two roundabouts, straight segments and pedestrian crossings at the entrance and exits of roundabouts. The vehicle is modeled with a dynamic bicycle model (as in [17]) and the whole scenario is simulated in MATLAB Simulink.

Initially, the vehicle is at full stop in point 1 of Fig. 4. The proposed destination is set at point 3 of Fig. 4, traveling through both roundabouts. Different controller configurations are evaluated in real-time and the resultant trajectories can be seen in blue in Fig. 5. Here, the road boundaries are depicted in red, the road middle path is given in orange, the most precise controller configuration is given in black and the most comfortable one is given in magenta.

An example of different controllers' responses can be seen in Fig. 6. The lateral errors for the different cases are given in Fig. 6a, where the controller tracking the trajectory as close as possible is presented in black, showing a maximum lateral error below $0.2 \mathrm{~m}$; and the smoothest controller shows lateral deviations close to $1.5 \mathrm{~m}$ (still within the lane boundaries). Intermediate controller responses are depicted in blue. 


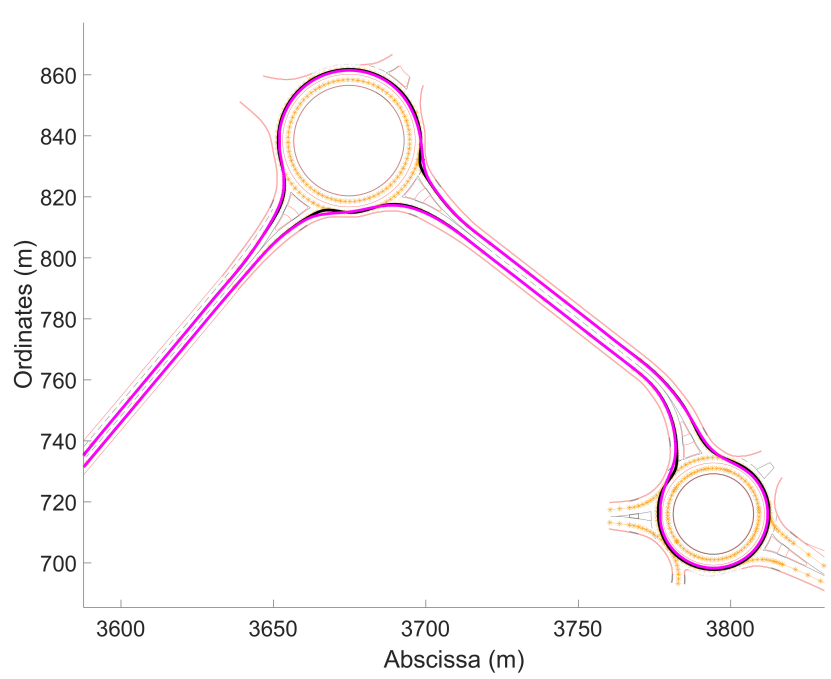

Fig. 7: Bird view of the real scenario.

The steering wheel angle and the steering wheel angle rate are presented in Fig. 6b and Fig. 6c. The controllers' response are presented in black, magenta and blue for the tight, loose and intermediate controllers respectively. Figure 6 shows that, even if lateral displacement increases when implementing the loose controller, the vehicle needs less important steering actions. This decreases both the steering wheel maximum angle needed to turn and the steering rate, providing smaller lateral accelerations and increasing comfort. Any of the controllers allow the vehicle to come back to the center of the lane when in straight segments, ensuring also a proper behavior in these scenarios.

\section{SYSTEM VALIDATION}

The proposed system has been tested in real scenarios. The experimental platform is a computer-controlled Renault ZOE. The vehicle is completely robotized, enabling the control of the steering wheel, throttle and brake pedals. For the sake of clarity, the tests were carried out using a precise positioning system and no traffic interaction. This allow to isolate the proposed control architecture for any failures from other modules that can impact system's evaluation.

Experimental tests were carried out at Rambouillet Territories, France. Fig. 7 shows the whole scenario with the two different controller configurations (tight and loose). The zoom-in Fig. 8 allows to see the performance of the two paths taken by the real vehicle, where the loose controller (in magenta) diverts considerably more from the trajectory than the tight one (in black), giving a more human-like response while staying within road boundaries.

Figure 9a depicts the lateral error through the whole path. The tight controller achieves lateral errors under $0.2 \mathrm{~m}$, while the loose configuration presents lateral errors close to $1.5 \mathrm{~m}$. The latter smooths the trajectory the vehicle will follow as it increases comfort and reduces lateral accelerations, while always keeping the vehicle within bounds.

Figure 9b shows the steering wheel angle described in both runs. It is visible that the more the controller tries to precisely follow the path, the higher is the required steering wheel angle. In contrast, the loose controller prevents hard changes in the steering wheel angle, avoiding jerky behaviors, improving comfort.

Fig. 9 also reveals that the vehicle implementing the loose controller configuration gains a small amount of time while travelling through the path. As a matter of fact, this is visible as signals in Fig. 9b are somewhat synchronized at the beginning of the test (around second 60) and the temporal gain of the loose controller becomes more clear through time (see second 170) when synchronization is lost.

\section{CONCLUSIONS}

Comfort and safety are key design factors for the acceptance of driverless shuttles technology. This paper proposed a novel way of designing the control algorithm combining the closed loop system bandwidth and the future vehicle prediction states. This allows to smooth vehicle response whereas it remains within the lane boundaries. Since the definition of comfort and users' perception of safety change from user to user [14], the design allows the user adapt to his/her preference the vehicle response.

This permits to establish a link between the control design and the passenger, which is completely oriented to users' acceptance. Safety is ensured by computing the different controller configurations and ensuring the vehicle is always within the lane. Results show how the trajectory tracking can be either accurate, relaxed up to the lane boundaries or any intermediate configuration, profiting from the full lane width and increasing comfort without compromising safety.

The vehicle is able to travel within different scenarios such as roundabouts, pedestrian crossings, straight stretches, among others. The tests provided good results in simulations and in real tests, showing that, even if the vehicle sacrifices trajectory tracking performance, the gain in smoothness does not compromise the safety. It also reduces the steering wheel angle and steering wheel angle rate to take over turns and roundabouts, while also preserving a proper behavior in straight stretches. This translates into less lateral accelerations, shorter travels, higher passenger comfort and a smooth navigation, where all this is oriented towards increasing passenger acceptance.

Future works will focus on including other road users. This will inform the control block of the possibility to adopt tight or loose trajectory following behaviors when obstacles are present within the lane boundaries area. Also, future experiments are foreseen to thoroughly test our approach and assess passengers' feedback in terms of comfort, safety, ride quality and other different aspects; integrating these in the vehicle motion planning and control design strategy.

\section{ACKNOWLEDGMENT}

Authors thank FUI Tornado project to partially fund this work. Authors want also express their gratitude to the Rambouillet Territoire, and especially to Carole Forte for the support on the testing preparation. The authors wants to also thank the RENAULT Research Department for its support in developing experimental tests. 


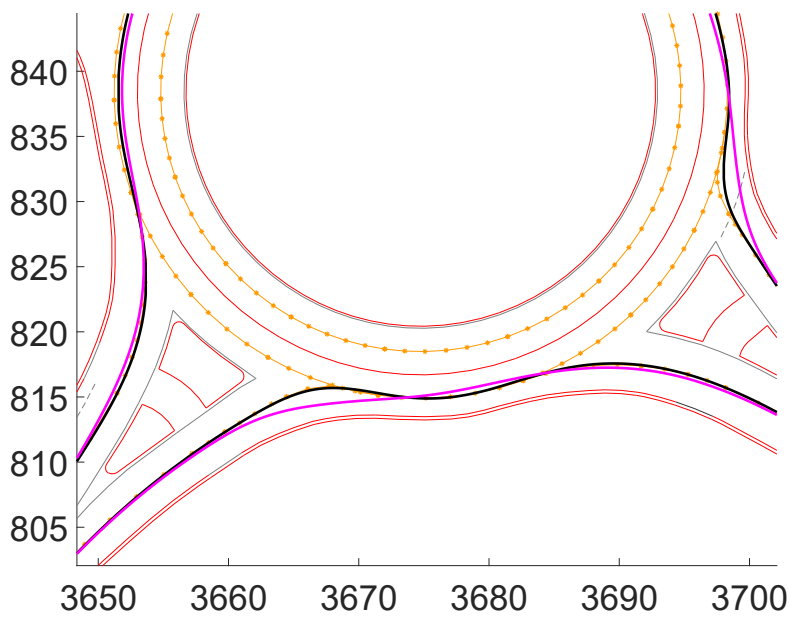

(a) Zoom, first roundabout

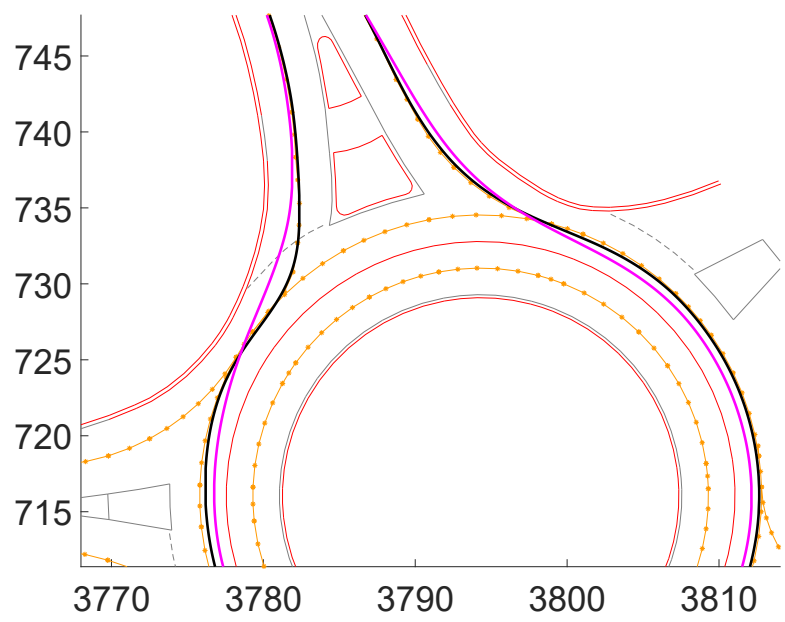

(b) Zoom, second roundabout

Fig. 8: Zoom-in of the two roundabouts in the real scenario. Boundaries are depicted in red and the road's center-line is shown in orange. Both tight and loose controllers are tested, presented in blue and magenta respectively.

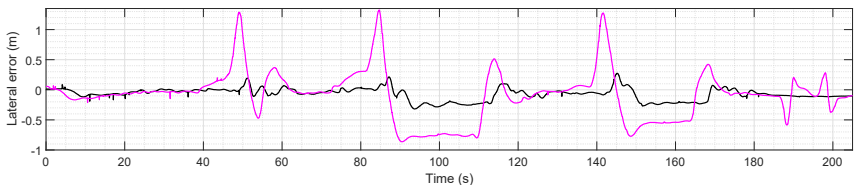

(a) Lateral error

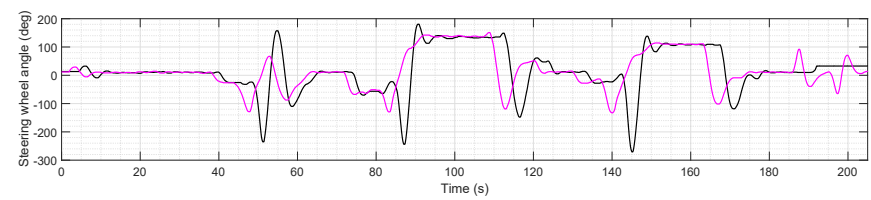

(b) Steering wheel angle response

Fig. 9: Lateral error and steering wheel angle response for both controller configurations (tight and loose). The most restrictive controller configuration is depicted in black (tight) and the most relaxed one is presented in magenta (loose).

\section{REFERENCES}

[1] S. Kim, G. Gwon, W. Hur, D. Hyeon, D. Kim, S. Kim, D. Kye, S. Lee, S. Lee, M. Shin, and S. Seo, "Autonomous campus mobility services using driverless taxi," IEEE Trans. Intelligent Transportation Systems, vol. 18, no. 12, pp. 3513-3526, 2017. [Online]. Available: https://doi.org/10.1109/TITS.2017.2739127

[2] V. Milanés, J. Alonso, L. Bouraoui, and J. Ploeg, "Cooperative maneuvering in close environments among cybercars and dual-mode cars," IEEE Transactions on Intelligent Transportation Systems, vol. 12, no. 1, pp. 15-24, 2011.

[3] K. Malone, J. van der Wiel, and B. Saugy, "Cybernetic transport systems: lessons to be learned from user needs analysis and field experience," in Intelligent Vehicle Symposium, 2002. IEEE, vol. 2, June 2002, pp. 551-556 vol.2.

[4] J. van Dijke and M. van Schijndel, "Citymobil, advanced transport for the urban environment: Update," Transportation Research Record: Journal of the Transportation Research Board, vol. 2324, no. 2324, pp. 29-36, 2012.

[5] J. Dokic, B. Müller, and G. Meyer, "European roadmap smart systems for automated driving," European Technology Platform on Smart Systems Integration, 2015.
[6] R. Madigan, T. L. Louw, M. Wilbrink, A. Schieben, and N. Merat, "What influences the decision to use automated public transport? using utaut to understand public acceptance of automated road transport system," Transportation Research Part F: Traffic Psychology and Behaviour, vol. 50, pp. 55-64, 2017.

[7] Y. Du, C. Liu, and Y. Li, "Velocity control strategies to improve automated vehicle driving comfort," IEEE Intelligent Transportation Systems Magazine, vol. 10, no. 1, pp. 8-18, 2018.

[8] D. González, V. Milanés, J. Pérez, and F. Nashashibi, "Speed profile generation based on quintic bézier curves for enhanced passenger comfort," in 2016 IEEE 19th International Conference on Intelligent Transportation Systems (ITSC), Nov 2016, pp. 814-819.

[9] C. Liu, W. Zhan, and M. Tomizuka, "Speed profile planning in dynamic environments via temporal optimization," in Intelligent Vehicles Symposium (IV), 2017 IEEE. IEEE, 2017, pp. 154-159.

[10] S. Lefèvre, A. Carvalho, and F. Borrelli, "A learning-based framework for velocity control in autonomous driving," IEEE Transactions on Automation Science and Engineering, vol. 13, no. 1, pp. 32-42, 2015.

[11] M. Kuderer, S. Gulati, and W. Burgard, "Learning driving styles for autonomous vehicles from demonstration," in 2015 IEEE International Conference on Robotics and Automation (ICRA). IEEE, 2015, pp. 2641-2646.

[12] J. M. Anderson, K. Nidhi, K. D. Stanley, P. Sorensen, C. Samaras, and O. A. Oluwatola, Autonomous vehicle technology: A guide for policymakers. Rand Corporation, 2014.

[13] D. González, J. Pérez, V. Milanés, and F. Nashashibi, "A review of motion planning techniques for automated vehicles," IEEE Transactions on Intelligent Transportation Systems, vol. 17, no. 4, pp. 1135-1145, April 2016.

[14] H. Bellem, B. Thiel, M. Schrauf, and J. F. Krems, "Comfort in automated driving: An analysis of preferences for different automated driving styles and their dependence on personality traits," Transportation research part F: traffic psychology and behaviour, vol. 55, pp. 90-100, 2018.

[15] M. V. P. J. Villagra, J. and J. Godoy, "Smooth path and speed planning for an automated public transport vehicle," Robotics and Autonomous Systems, vol. 60, no. 2, pp. 252-265, 2012.

[16] I. Mahtout, F. Navas, D. Gonzalez, V. Milanés, and F. Nashashibi, "Youla-Kucera Based Lateral Controller for Autonomous Vehicle," in ITSC 2018 - 21st IEEE International Conference on Intelligent Transportation Systems, Maui, Hawaii, U.S. Outlying Islands, Nov. 2018. [Online]. Available: https://hal.inria.fr/hal-01906268

[17] R. Rajamani, Vehicle dynamics and control. Springer Science \& Business Media, 2011.

[18] S. M. LaValle, Planning algorithms. Cambridge university press, 2006. 